\title{
Psychologie cognitive et production écrite : de l'effet « épistémique » à la catalyse
}

\section{Sabine Pétillon}

\section{(2) OpenEdition}

1 Journals

Édition électronique

URL : https://journals.openedition.org/genesis/141

DOI : $10.4000 /$ genesis. 141

ISSN : 2268-1590

Éditeur :

Presses universitaires de Paris Sorbonne (PUPS), Société internationale de génétique artistique littéraire et scientifique (SIGALES)

Édition imprimée

Date de publication : 20 juin 2010

Pagination : 197-206

ISBN : 978-2-84050-697-3

ISSN : 1167-5101

\section{Référence électronique}

Sabine Pétillon, "Psychologie cognitive et production écrite : de l'effet « épistémique » à la catalyse », Genesis [En ligne], 30 | 2010, mis en ligne le 30 mai 2012, consulté le 30 mars 2023. URL : http:// journals.openedition.org/genesis/141 ; DOI : https://doi.org/10.4000/genesis.141 


\title{
Psychologie cognitive et production écrite : de l'effet « épistémique » à la catalyse
}

\author{
Sabine Pétillon
}

$\mathrm{D}$ ans le cadre de ce travail et des limites qui lui sont imparties, notre objectif est de proposer une présentation sommaire de quelques concepts majeurs en psychologie de la production écrite afin d'examiner comment ces concepts - notamment celui de processus, d'effet épistémique et de signature rédactionnelle - peuvent être exportés vers les manuscrits de travail des écrivains. Après avoir exposé quelquesuns des acquis majeurs des modèles rédactionnels, nous proposons une analyse processuelle de la digression dans le manuscrit de Langage Tangage de Michel Leiris, afin de voir en quoi il peut illustrer certains éléments de l'approche de l'écriture proposée en psychologie. Ce faisant, en filigrane - sur un plan tout à la fois historique et méthodologique -, nous proposons de mettre à jour les points de rencontre et les divergences entre la critique génétique et la psychologie cognitive. Cette mise en perspective voudrait éclairer les possibilités de collaboration à venir ${ }^{1}$.

\section{Modèles rédactionnels et dossier de genèse : un matériau commun?}

Depuis plus de vingt ans, la psychologie cognitive s'est consacrée non seulement à la compréhension mais aussi à la production des textes : c'est cette spécificité des travaux portant sur la production écrite que nous souhaitons présenter d'abord. On peut commencer par une définition : «La production de textes est souvent définie, dans le cadre de la psychologie cognitive, comme une activité mentale complexe supposant la mise en œuvre d'un ensemble de connaissances langagières et de différents processus mentaux ${ }^{2}$. »

Depuis les années quatre-vingt, plusieurs modélisations de la production écrite ont été proposées par des psychologues américains, J.-R. Hayes et L. Flower (1980), puis, notamment pour l'écriture experte, par C. Bereiter et M. Scardamalia (1987). Plus spécifiquement, c'est à l'étude de la production de textes courts (mémoires universitaires, rapports, textes juridiques, articles de journaux et textes procéduraux ${ }^{3}$ ), produits par des étudiants ou des rédacteurs professionnels, que les psychologues se sont attachés. De nombreuses études ont également porté sur l'écriture collaborative au cours de l'acquisition de l'écriture, mais aussi en milieu professionnel. Les corpus étudiés en psychologie de la production sont donc constitués de textes ordinaires et l'objectif - qu'il s'agisse de milieux professionnels ou scolaires - est de fournir des analyses susceptibles d'apporter une amélioration de la production des scripteurs. Dans cette perspective, les psychologues ont décrit l'écriture comme un processus terme central, et commun avec la critique génétique 4 - que l'on peut analyser de façon plus précise en

1. Je tiens à remercier vivement Anne Herschberg Pierrot pour l'aide précieuse qu'elle m'a apportée dans l'élaboration de ce travail.

2. D. Alamargot et L. Chanquoy, « Les modèles de rédaction de textes », Production du langage, dir. M. Fayol, Paris, Hermes, 2002, p. 45-65, p. 45. Dans ce même volume, on se reportera également avec profit à J.-L. Lebrave, «Critique génétique et processus d'écriture littéraires », p. 251-261. Voir aussi D. Alamargot et L. Chanquoy, Through the Models of Writing, Studies in writing, vol. IX, Dordrech, Boston, London, Kluwer Academia Publishers, 2001.

3. Sur les textes procéduraux, voir notamment, F. Ganier, « La révision de textes procéduraux », Langages, $n^{\circ} 164,2006$, dir. S. Pétillon et F. Ganier, p. 71-85.

4. En évoquant la distinction entre l'écriture à programme et l'écriture à déclenchement rédactionnel, P.-M. de Biasi insiste sur la position centrale du processus : «Ces deux genèses obéissent l'une et l'autre aux différents processus (exploratoire, documentaire, rédactionnel, etc.) qui leur sont communs. La notion de processus, évidemment centrale en génétique, doit être réservée à un usage terminologique approprié ", La Génétique des textes, Paris, Nathan, coll. « $128 », 2000$, p. 33. 
termes de sous-processus et d'enchaînements de sousprocessus. Le degré d'aisance, dans la manipulation de ces processus et dans leur interaction, a permis à la psychologie cognitive d'étudier et de typologiser les productions écrites de scripteurs très divers, tant au plan du niveau d'expertise (distinction novice/expert) qu'au plan de la difficulté du texte à produire (textes techniques/écrits créatifs).

L'observation de ces différents textes et scripteurs a donc permis à la psychologie de distinguer trois sousprocessus dans le processus scripturaire :

1) La planification qui permet d'élaborer, après avoir déterminé les arguments/thème, un plan guidant l'exécution du processus rédactionnel dans son ensemble : la planification prépare donc la rédaction à venir. L'une des étapes préliminaires de la planification est de déterminer le but du texte - j'écris pour quoi faire, pour dire quoi, à qui ? - et donc le genre discursif dans lequel intervient l'écriture : essai, texte argumentatif, notice d'utilisation, genres journalistiques ${ }^{5}$. Dans ce moment de planification, le scripteur met en œuvre toutes sortes d'activités cognitives et tout particulièrement des opérations d'activation-sélection des connaissances et des contenus et des opérations d'organisation-composition: c'est cet ensemble complexe d'opérations préparatoires que l'on appelle la planification. Elles font intervenir la mémoire de travail à long terme. Ces opérations se concrétisent plutôt sous la forme de segments langagiers n'ayant pas la forme de phrases complètes, comme on peut le voir aussi dans les manuscrits d'écrivains.

2) La textualisation - que l'on appelle aussi « rédaction » - met en œuvre des opérations relevant non plus du niveau général de la structuration d'un plan, mais du plan local. Dans ce plan local, la détermination devient spécifique, la chronologisation intervient grâce aux tiroirs verbaux, la syntaxe se complexifie. C'est dans ce moment de la texualisation que l'on peut observer des profils tendanciels de scripteurs : les uns ayant tendance à réduire une première textualisation (réduction du volume textuel), les autres choisissant plutôt d'épaissir leur première version à coups d'ajouts (amplification du volume textuel). C'est du reste la distinction que Barthes a opérée en distinguant les « esprits à ellipse » et les « esprits à catalyse » dans son célèbre article "Flaubert et la phrase », que nous sommes actuellement en train d'illustrer grâce à différents dossiers de genèse 6 . La textualisation intervient donc au plan phrastique mais aussi au plan de la mise en forme matérielle du texte -notamment de la matérialisation du texte dans l'espace du paragraphe et de la page 7 .

3) Enfin la révision se définit comme l'ensemble des retouches, corrections opérées sur le texte en cours. Il s'agit en général - pour ce que l'approche psychologique a pu décrire - de corrections locales plus que de remaniements conduisant à un changement du matériau déjà écrit.

L'activation de ces trois sous-processus - qui fonctionnent de façon plus ou moins concomitante - engendre des types de documents hétérogènes : notes, plans, plans semirédigés, synthèses, rapports, mémoires, essais textualisés. Autant de types de documents qui ont été également mis au jour - dans les mêmes années quatre-vingt - et typologisés par la critique génétique dont la définition des phases rédactionnelles rappelle les trois processus établis par la psychologie.

En effet, sans revenir sur le détail, on rappellera très brièvement, pour mémoire, la notion de phase rédactionnelle (ou phase créative) établie par P.-M. de Biasi - notion qui permet le classement des types de documents de genèse selon leur fonctionnalité et la reconstitution de la genèse de l'œuvre. P.-M. de Biasi a en effet distingué d'abord la phase prérédactionnelle qui correspond aux moments préparatoires de l'œuvre. Les documents génétiques qui relèvent de cette phase sont, comme on le sait, très variés : listes, titres, notes

5. Sur la question des genres (premiers, relevant de l'écriture ordinaire, ou seconds, relevant de la littérature), voir l'excellente typologie proposée par D. Maingueneau, « Modes de généricité et compétence générique », La Licorne, $\mathrm{n}^{\circ} 79$, « Le savoir des genres », 2006, p. 57-71.

6. Voir S. Bikialo et S. Pétillon, « La phrase et le style : des invariants processuels à la variance individuelle », Pratiques, $\mathrm{n}^{\circ} 135-136$, « Pratiques des styles », 2007, p. 170-192.

7. Sur la mise en forme matérielle du texte, voir les travaux du psychologue W. Kintsch, mais aussi au plan de la linguistique textuelle, ceux de J.-M. Adam (notamment pour ce qui concerne la schématisation, c'està-dire la co-construction d'un texte comme « résultat » et comme « dynamique », par les co-énonciateurs). J.-M. Adam, Linguistique textuelle. Des genres de discours aux textes, Paris, Nathan, 1999, et J.-M. Adam, La Linguistique textuelle. Introduction à l'analyse textuelle des discours, Paris, Armand Colin, 2005. Voir également A. Rey, «Tracés », dans L. Hay (dir.), De la lettre au livre. Sémiotique du manuscrit littéraire, Paris, CNRS Éditions, coll. « Textes et Manuscrits », 1989. 
documentaires, plans ou plans développés sous forme de scénarios, liste de mots, liste dialogique de questions ou de directives sur la rédaction à venir, etc. Ces documents manuscrits - qui relèvent, dans une large mesure, de la génétique scénarique - sont le lieu d'établissement d'un contenu informatif, d'un choix référentiel : ils permettent au scripteur de construire un univers scripturaire dans lequel s'élabore une représentation discursive. On retrouve bien là ce qui est décrit en psychologie. La phase rédactionnelle permet au généticien de repérer les modes d'ancrage syntaxique des segments langagiers intervenus, dans la phase préparatoire, sous forme de notes, de cellules langagières. S'observe également dans ce moment-là, l'entrée de la structure phrastique dans une dynamique de complexification en fonction d'une progression thématique précise. Pour reconstituer les processus qui ont présidé

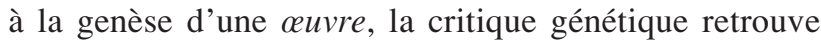
donc, pour une part, la typologie documentaire que les modèles rédactionnels observent dans la production de textes ordinaires. Ainsi, il est étonnant de constater que la psychologie de la production verbale écrite et la critique génétique ne sont pas encore vraiment parvenues à collaborer, alors même qu'elles ont en commun au moins deux orientations heuristiques : l'analyse des processus d'écriture et la mise à jour d'une typologie de documents constitués en objets d'études de ces différents processus. Si l'objet d'investigation est le même - les processus d'écriture -, la psychologie n'a pas étudié la production littéraire, se privant ainsi d'un des lieux d'écriture les plus complexes qui soient. De même, la critique génétique a peu approché les outils de la psychologie de la production. Nous proposons donc, maintenant que nous avons décrit succinctement la modélisation des processus rédactionnels, d'examiner comment certains des outils et des méthodes de la psychologie pourraient être exportés - ou pas - vers la critique génétique.

\section{Modes d'écriture, effet épistémique et définition processuelle du style}

Au-delà des frontières institutionnelles et idéologiques (distinction textelouvre), le peu de collaborations entre psychologues et généticiens vient sans doute d'abord de l'objet d'étude : les psychologues travaillent sur les processus d'écriture (ou de compréhension) de tous types de textes et l'objectif est d'analyser les opérations mentales complexes que l'activité de langage permet de mettre au jour. Par ailleurs, jusqu'à une date récente, la question de la création et de l'œuvre était relativement étrangère à l'approche cognitive, et l'objectif de la modélisation de la production verbale restait, avant tout, d'améliorer la qualité du texte écrit. Cet objectif n'a jamais été celui de la génétique, qui, jusqu'ici, s'est intéressée, certes au processus, mais dans le cadre de la genèse des $œ u v r e s$ et du littéraire (sans visée méliorative bien évidemment ${ }^{8}$ ). De plus, les méthodes d'investigation diffèrent : l'approche méthodologique de la psychologie autorise l'expérimentation, la manipulation des corpus, et le guidage du recueil des données chez les informants - ce qui ne peut évidemment pas être le cas de la génétique. À la constitution de groupes suffisants pour valider l'interprétation de résultats qui interviennent souvent sous forme de pourcentage et de schémas divers s'oppose l'irréductible singularité de l'écrivain. D'un côté les écrivains, de l'autre les écrivants. Vu sous cet angle, on comprend aisément le no man's land qui sépare les deux disciplines et le grand écart qu'implique la tentation de les relier ! C'est du reste ce que souligne, à regret, D. Alamargot : « À l'heure actuelle, la psychologie cognitive ne semble pas encore avoir entrepris d'études systématiques auprès d'écrivains ${ }^{9} \gg$ pour décrire le fonctionnement de stratégies rédactionnelles qui

\footnotetext{
8. Même si l'imitation des grands écrivains a pu constituer un exercice majeur des classes de rhétorique, exercice que l'on retrouve aujourd'hui dans l'écriture d'invention - à partir du texte d'un auteur - du programme des lycées. La démarche d'A. Albalat, au début du Xx $x^{\mathrm{e}}$ siècle, constitue à ce titre un moment important de cette écriture imitative. Voir A. Albalat, La Formation du style par l'assimilation des auteurs, Paris, Armand Colin, 1934.

9. Voir S. Pétillon, «Style, critique génétique et modèles rédactionnels : perspectives linguistiques », Corpus, $\mathrm{n}^{\circ}$ 5, décembre 2006 ; D. Alamargot, «Du rédacteur à l'écrivain : point de vue de la psychologie cognitive », dans Critique génétique. Concepts, méthodes, outils, dir. O. Anokhina et S. Pétillon, Paris, IMEC-éditeur, coll. «Inventaires », 2009, p. 160-171, p. 166 ; voir également I. Fenoglio et J.-M. Adam (dir.), Modèles linguistiques, vol. XXX, $\mathrm{n}^{\circ} 59$, « Génétique de la production écrite et linguistique », 2009.
} 
semblent, comme le montrent les manuscrits, bien plus complexes que celles qui guident les novices ou même les rédacteurs experts. Aux yeux du psychologue, la production de l'hyper expert qu'est l'écrivain apparaît donc bien comme un nouvel eldorado de l'activité mentale. Mieux, comme une nouvelle frontière scientifique - infranchissable?

Sans doute pas. Et nous voudrions esquisser ici deux pistes communes : les modes d'élaboration scripturaires (qui touchent au traitement des contenus) et la signature rédactionnelle (qui a trait à l'enchaînement des sousprocessus), et qui rejoint du reste la question des styles de genèse proposée récemment par A. Herschberg Pierrot ${ }^{10}$.

\section{Les deux modes d'élaboration scripturaire en psychologie : croisements avec le manuscrit littéraire}

Comme nous l'avons déjà souligné, dès les années quatre-vingt, au moment de son émergence, le domaine d'investigation de la psychologie de la production le « corpus »- a été constitué de productions issues d'élèves ou d'étudiants en cours de formation : l'analyse de ces productions s'est donc faite dans une perspective acquisitionnelle et didactique. Par ailleurs, depuis les années quatre-vingt-dix, l'écriture experte, c'est-à-dire la production de scripteurs confirmés, a été principalement analysée à travers des textes de presse, des rapports techniques, des guides divers, des lettres commerciales et - tout récemment - à travers les textes procéduraux. L'analyse de l'écriture experte a permis de montrer des différences notables - dans la stratégie et l'enchaînement des processus - en fonction du type de document produit et de son cahier des charges. Les scripteurs experts pratiquant un genre spécifique et fortement contextualisé stabilisent très fortement les processus qu'ils mettent en œuvre : tous les écrits techniques (lettres type, modes d'emploi, etc.) impliquent un plan, un lexique, et bien sûr une syntaxe spécifique, mais l'ensemble est peu soumis à variation. Ces écrits interviennent dans une configuration de problèmes relativement fermée. Le degré de liberté de la tâche est assez réduit - ce qui autorise un contrôle de l'ensemble de l'activité et de ses niveaux : planification/textualisation; plan macro/microstructurel. Le scripteur construit un plan toujours plus ou moins identique, et un texte univoque sur le plan générique : on observe une stabilisation des stratégies, c'est-à-dire une succession prévisible entre la planification, la rédaction puis la révision. En revanche, l'étude de la production de textes non littéraires mais non techniques, c'est-à-dire impliquant une part de créativité a permis d'observer des stratégies rédactionnelles plus élaborées dont nous rendrons compte ici en suivant les données établies par D. Alamargot 11 .

Dans sa présentation des modes d'élaboration scripturaire, D. Alamargot rappelle la distinction opérée notamment par le psychologue D. Galbraith (1990) entre le mode «classique » et le mode "romantique » d'écriture ${ }^{12}$. Relève du mode dit "classique » (schemadriven), le texte qui s'élabore d'abord à partir d'un plan de travail, et qui détermine donc des contenus préalables. C'est nécessairement le cas pour les écrits techniques que nous avons évoqués : lettre commerciale, notices d'utilisation, rapports divers. Ces textes impliquent un schéma préétabli et réduisent grandement la créativité et l'invention pendant la rédaction. On notera ici - car il faudrait croiser les modes d'écriture et les genres de discours - que ces textes croisent la typologie des genres discursifs établie en analyse du discours, de façon très pertinente, par D. Maingueneau ${ }^{13}$. Au contraire, le mode « romantique » (text-driven) opère par rédaction libre, sans plan préalable. Dans ce dernier cas, le scripteur alterne une phase de libre expression de la pensée dans un flux d'écriture continue (sans révision) et une phase d'évaluation critique de cette production. On insistera ici sur l'idée que le mode "romantique » ou " classique » n'est qu'un étiquetage de processus, et que ces deux termes - romantique/classique - ne qualifient en rien un

10. A. Herschberg Pierrot, Le Style en mouvement. Littérature et art, Paris, Belin, coll. « Lettres », 2005, p. 141-172.

11. D. Alamargot, « Du rédacteur à l'écrivain... », art. cit.

12. D. Galbraith, "Conditions for discovery through writting », Instructional Science, $\mathrm{n}^{\circ} 21$, p. 45-72. Au-delà de la relative maladresse dans le choix de ces étiquettes relevant de l'histoire littéraire, cette distinction apparaît particulièrement opérante dans le champ génétique. 13. D. Maingueneau, «Retour sur une catégorie : le genre », dans Textes et discours : catégories pour l'analyse, dir. J.-M. Adam, J.-B. Grize et M. Ali Bouacha, Dijon, Éditions universitaires de Dijon, coll. «Langages », 2005, p. 107-118. 
auteur, un texte ou une œuvre14. Cependant, comme le souligne D. Alamargot : «Comparativement au mode "classique", le mode "romantique" a été encore très peu étudié par la psychologie cognitive. Plusieurs questions restent en suspens concernant : la nature des traitements impliqués dans la créativité, le contrôle des processus en l'absence de plan et l'interactivité des traitements pendant la rédaction 15 . » L'inexistence des travaux de psychologie sur la production des écrivains explique bien évidemment cette répartition entre d'une part les écrits techniques, qui relèvent du schema-driven, d'autre part l'écriture littéraire, qui relève du text-driven : il est évident que les textes littéraires peuvent également suivre un plan bien précis, et que celui-ci peut même évoluer en fonction de la textualisation ; cependant, la planification a certainement des incidences sur les processus de textualisation et de création (Flaubert, Valéry, Sartre). C'est la distinction, infiniment modulable, établie en critique génétique entre les écrivains à programme et les écrivains à déclenchement rédactionnel. On bute ici, de façon suggestive, sur ces pistes à ouvrir entre les deux disciplines. Et puisqu'il est ici question de piste, le mode d'écriture « romantique » (text-driven) nous en offre une avec la notion d'effet épistémique, sur laquelle D. Alamargot revient pour définir le mode « romantique16 ». Puisqu'il s'agit ici d'un dialogue interdisciplinaire, on peut rappeler la définition que donne P.-M. de Biasi de l'écriture des deux types d'écritures littéraires : « il y a des écrivains qui ne peuvent travailler qu'avec un canevas précis selon le principe d'une "programmation scénarique" qui anticipe sur la textualisation, et d'autres qui ont besoin de se jeter dans la rédaction sans se sentir contraints par le moindre plan en suivant la méthode d'une "structuration rédactionnelle" [...]. Cette opposition est essentielle car il s'agit de deux manières de travailler réellement différentes, et à certains points de vue opposées, qui ont des conséquences décisives sur la forme et la nature des manuscrits. L'écriture "à structuration rédactionnelle", qui est réfractaire à toute programmation initiale, ne s'appuie sur aucun schéma écrit, et va droit devant elle en commençant par une rédaction de "premier jet", qui avance en s'enrichissant de révisions à chaque relecture du déjà rédigé $[. .$.$] . Au contraire, l'écriture “à$ programmation scénarique" fait précéder l'écriture par un travail de conception préliminaire, sous la forme de plans, scénarios, notes, ébauches, notes documentaires, qui ont pour fonction de préparer et d'organiser une rédaction qui pourra ensuite être mise en œuvre partie par partie, chapitre par chapitre, page par page 17 [...]. »

\section{Au cour de la formulation: l'effet épistémique ou comment voir "s'élever le brouillard»?}

Privilégiant ce que la psychologie appelle l'« écriture libre », c'est-à-dire procédant sans plan, le mode « romantique » permet d'appréhender de façon spécifique la créativité et l'invention : celles-ci émergent en quelque sorte ex nihilo, offrant des dossiers de genèse sans plan préalable18. Comme le souligne D. Alamargot : "Le mode "romantique" est propice à l'invention des contenus. [Sans plan], le processus de formulation occupe une place centrale dans le modèle car ce serait le fait même d'élaborer le message écrit qui suscite la création d'idées 19 » (il faut sans doute considérer que c'est au moment de la textualisation - sans plan - que les contenus s'inventent, ce qui ne signifie aucunement que l'écriture scénarique soit rétive à l'invention !). Cette avancée du texte dans et grâce au «forage » (Benveniste) de la pensée dans les mots évoque à s'y méprendre les confidences d'un des plus fascinants scripteurs à déclenchement rédactionnel : Stendhal, qui confiait dans une lettre bien connue : « À chaque page je vois s'élever le brouillard qui couvrait la suivante » (28 mai 1839). Cet effet épistémique sous-jacent à l'écriture « libre » (l'adjectif « libre » vient de la psychologie) s'observe précisément dans les phases de recherche du mot juste : «Ce serait cette recherche du terme linguistique le plus adapté qui susciterait

14. Michel Leiris, et Langage Tangage, n'ont en effet rien de romantique. 15. D. Alamargot, «Du rédacteur à l'écrivain... », art. cit., p. 167. Nous soulignons.

16. Ibid., p. 168.

17. P.-M. de Biasi, La Génétique des textes, op. cit., p. 33.

18. On pourrait nuancer en soulignant que - pour ce qui est du mode d'écriture dit « classique »- la créativité et l'invention interviennent au niveau du plan et de l'élaboration des contenus préalables à leur mise en texte. Cela étant, la chronologie de l'invention est sans doute plus facile à reconstituer dans l'écriture sans plan.

19. D. Alamargot, « Du rédacteur à l'écrivain... », art. cit., p. 167. Nous soulignons. 
l'invention, par associations lexicales et sémantiques. Le fait de sélectionner un item lexical et de l'articuler avec les mots précédemment écrits active de nouveaux concepts, crée de nouveaux liens et de nouveaux contenus. L'intégration dans le texte de ces nouveaux éléments motive l'élaboration d'une nouvelle version, basée sur la révision de la précédente »(D. Alamargot). À titre d'illustration rapide, nous proposons maintenant l'analyse de quelques lignes brouillonnantes d'un autre grand écrivain à déclenchement rédactionnel : Michel Leiris.

Précisément, cette formulation continuellement inventive, cette incessante recherche du mot juste que constitue l'effet épistémique est particulièrement observable, en effet, dans la dynamique textualisante de la phrase leirissienne. Et la reconstitution de la genèse d'une phrase extraite du manuscrit de Langage Tangage permet, comme nous allons le voir, de mettre au jour un programme/un mécanisme syntaxique récursif d'expansion, au sens dynamique du terme, et d'intégration réitérée de "nouveaux contenus », pour reprendre la formulation de D. Alamargot. Dans notre microanalyse génétique, on peut isoler quatre moments d'écriture qu'il est possible de visualiser comme ceci : étape 1 (police) : chicago, étape 2 : courier, étape 3 : futura, étape 4 : geneva 20 .

\section{Objet d'une appréhension nauséeuse qui court en leitmotiv tout au long de ces feuillets, alors que je devrais m'abstenir en homme aussi bien élevé que son anglophilie de jeunesse et d'âge mûr le lui prescrit de faire vibrer à tout bout de champ la corde du pathétique, le saut qui nous fera franchir la rampe pour en fait ne nous mener nulle part est ce dont l'attente effrayée m'atterre et tout à la fois m'anime.}

Nous donnons ici, avec un énoncé de base relativement bref, une phrase-énoncé prototypique de la formulation leirissienne qui permet d'illustrer les mécanismes processuels d'un scripteur qui avoue construire ses phrases à coups d'" additifs » et de « rembourrages incessants 21 ». Avant d'entrer dans le détail de l'analyse, on soulignera que le dispositif d'écriture de Leiris - un premier jet au recto et des révisions au verso - anticipe le processus de révision dans une amplitude large : il est plus facile d'ajouter plusieurs phrases, et même une séquence entière, au verso d'un feuillet que dans l'interligne ou la marge. Toutes les retouches que nous analysons interviennent donc au verso.

$\mathrm{Si}$ nous revenons maintenant au détail de la formulation leirissienne, on observe que le processus d'insertion d'un élément nouveau - qui constitue la dynamique même de l'effet épistémique - intervient à l'intérieur du groupe nominal appositif en position frontale, rattaché au GN (« le saut») dont il constitue une expansion anticipatoire. Le premier insert, qui sert luimême de base pour d'autres ajouts à venir, est constitué d'une longue proposition temporelle en « alors que », qui s'achève sur cette impossibilité de ne pas «faire vibrer la corde du pathétique ». D'où l'amertume nauséeuse du portrait qui pourtant « en rajoute » dans les retouches pédantes. Et c'est le second mouvement des ajouts : viennent alors - dans cette proposition temporelle qui constituait le premier ajout - l'ajout d'une expansion caractérisante du nom « en homme »: l'adjectif «bien élevé » (structure comparative " aussi bien élevé que son anglophilie le lui prescrit»), et deux groupes prépositionnels coordonnés, « de jeunesse et d'âge mûr », expansions caractérisantes du nom « anglophilie », et c'est le troisième temps d'ajout/expansion processuel. Le texte, ici, c'est-à-dire au seuil de la mort, ne se désolidarise pas du genre autobiographique, de l'autoportait qui (re) surgit, de façon insistante, dans la genèse même du texte : au moment même de mourir, Leiris ne cesse pas de se décrire - et de retoucher son portrait, par le menu. L'accumulation des expansions adjectivales brèves met en valeur cette incessante retouche du trait. Leiris, comme

20. Il s'agit des feuillets $111 \mathrm{r}^{\circ}, 124 \mathrm{v}^{\circ}$ et $111 \mathrm{bis} \mathrm{r}^{\circ}$ de l'état manuscrit de Langage Tangage (LRS Ms 108), qui se trouvent à la Bibliothèque littéraire Jacques Doucet.

21. M. Leiris, Langage Tangage ou Ce que les mots me disent, Paris, Gallimard, coll. «L'imaginaire », 1995, p. 111. Dans Biffures, Leiris confie également : «Je ne puis m'empêcher d'exprimer ma pensée sous forme de période, de groupes de propositions toujours plus ou moins cadencées » (Paris, Gallimard, coll. "L'imaginaire », p. 84). Sur cet itinéraire, voir également, J. Lambilliotte, « Michel Leiris : genèse d'une "formation parasitaire" dans un fragment de $\grave{A}$ cor et à cri », Langages, $\mathrm{n}^{\circ} 147$, «Processus d'écriture et marques linguistiques », dir. I. Fenoglio et S. Pétillon, 2000, p. 85-97. 
d'autres, mais à sa manière, suit les méandres génétiques de cette phrase longue qu'il pratique. Ce faisant, il illustre pleinement ce que Barthes appelle, à la suite de Hjelmslev, la catalyse : «la phrase peut être pourvue à l'infini d'incises et d'expansion : le travail catalytique est théoriquement infini [et] l'écrivain, affronté à la phrase, éprouve la liberté infinie de la parole, telle qu'elle est inscrite dans la structure même du langage $22 »$.

Cette « liberté infinie de la parole », également évoquée par Benveniste, pourrait être interrogée de façon plus précise par l'approche génétique. Autrement dit mais cela demanderait de plus amples investigations -, la textualisation fonctionne probablement suivant des modalités différentes, selon qu'elle suit un scénario, un schéma préétabli ou selon qu'elle s'invente un contenu en s'écrivant. Pour creuser les mécanismes linguistiques de cette distinction entre une écriture qui suit un plan préétabli ou qui s'invente en écrivant, il faudrait sans doute envisager le dossier de genèse à l'aune de la question de la schématisation telle qu'elle a été posée par J.-M. Adam : l'existence de plans préétablis de textes, dans l'interdiscours, doit être croisée avec les processus de textualisation ${ }^{23}$.

\section{Style de genèse et signature rédactionnelle}

Comme nous venons de le voir, l'analyse des manuscrits de Leiris permet de montrer que certains termes (présents dès la première étape de rédaction et jusqu'au texte définitif) jouent le rôle de repère pour l'écriture : leur statut de pivot est à la fois syntaxique (leur fonction grammaticale fonde la relation prédicative) et sémantique. Comme on a pu l'observer, ces segments phrastiques minimaux sont peu à peu séparés par des ajouts de nature grammaticale diverse, mais ils témoignent d'un même style de genèse. C'est cette notion de style de genèse, établie par A. Herschberg Pierrot, que nous voudrions, à l'aide de l'exemple de Leiris, rapprocher de celle de signature rédactionnelle décrite notamment par les psychologues J. R. Hays et L. Flower ${ }^{24}$.

Dans une approche stylistique, A. Herschberg Pierrot définit et illustre la notion de style de genèse comme ceci : «L'hétérogénéité des styles dans la genèse peut correspondre à une diversité de manières essayées 25 . » Ou encore : «On peut esquisser l'idée de styles de genèse. Ce serait, au-delà des irréductibilités liées à l'unicité du manuscrit, souligner des profils de genèse, dont participeraient les écrivains et leurs manuscrits, et qui seraient relatifs à la combinatoire d'une pratique d'écriture et de la réalisation d'une œuvre singulière 26 . » Et en effet, comme on l'observe dans les manuscrits, ces manières d'écrire peuvent être très différentes les unes des autres 27 . On pourra distinguer, comme le souligne A. Herschberg Pierrot, plusieurs types de scripteurs : des écrivains comme Beckett, Michon ou Bernard Noël qui pratiquent la rature blanche - ces écrivains ne corrigent jamais un premier état, mais le recopient (second temps) pour le corriger : la rature n'apparaît pas, mais s'observe en comparant les deux états ; des écrivains de premier jet (comme Mauriac, qui corrige au fil de la plume) ; et des écrivains qui font «d'emblée d'intenses réécritures et relectures, comme Flaubert 28 » ou Proust, ou Leiris et Claude Simon. Autrement dit, les uns rédigent d'abord l'intégralité de leur texte, puis le corrigent (ce que l'on décrit comme un processus sériel en psychologie), les autres l'écrivent tout en ne cessant de le réviser (processus concomitants : il y a tout à la fois rédaction et révision).

En viendrait-on à lier ces différents styles de genèse avec la qualité du texte final ? Une réponse positive ferait un lien provocateur mais incitatif vers la psychologie de la production écrite - et la notion de signature rédactionnelle. Cette signature est présentée

22. R. Barthes, « Flaubert et la phrase », Nouveaux Essais critiques, Paris, Éditions du Seuil, 1967, p. 135. Les recherches de Barthes autour de la phrase ont donné lieu à un séminaire (2002-2004) de l'équipe Manuscrit et Linguistique de l'ITEM alors dirigée par A. Grésillon.

23. J.-M. Adam, Linguistique textuelle. Des genres de discours aux textes, Paris, Nathan Université, coll. «Fac », 2004, p. 68-78.

24. J. R. Hays, L. S. Flower, « Uncovering cognitive processes in wrinting: an introduction of protocol analysis », Research on writing: principles and methods, New York, London, Longman, 1986.

25. A. Herschberg Pierrot, Le Style en mouvement, op. cit., p. 102.

26. A. Herschberg Pierrot, "Style et genèse des œuvres », Pratiques, $\mathrm{n}^{\circ} 135 / 136,2007$, p. 163-176. Nous soulignons.

27. Et varier en fonction du genre discursif. Par exemple, pour Sartre chez qui l'on observe des documents de genèse différents : l'écriture de premier jet des carnets (écriture philosophique) et l'écriture littéraire, beaucoup plus brouillonnée (voir M. Contat et J. Deguy, « Les carnets de la drôle de guerre de Jean-Paul Sartre. Effets d'écriture, effets de lecture », Littérature, $\mathrm{n}^{\circ} 80,1990$, p. 17-41).

28. A. Herschberg Pierrot, Le Style en mouvement, op. cit., p. 76. 
par D. Alamargot (sur la base bien sûr des travaux des psychologues américains) comme «l'analyse des probabilités de transition entre les processus qui montre que les enchaînements diffèrent selon les rédacteurs, mais sont relativement stables pour un rédacteur donné. Cette stabilité est interprétée comme l'empreinte du rédacteur, sa signature rédactionnelle (writing signature) $29 »$. Cette relative stabilité dans l'enchaînement des sous-processus d'écriture se trouve assez bien illustrée par les manières d'écrire - les styles de genèse que décrit A. Herschberg Pierrot - que les dossiers de genèse permettent d'observer : on tient là - incontestablement - une piste, un terrain d'investigation commune entre la génétique et la psychologie de la production.

En effet, l'hypothèse de l'existence d'enchaînements de processus privilégiés selon les rédacteurs doit d'abord être vérifiée (et consolidée auprès des écrivains) : elle est très certainement à l'origine de cette reconnaissance intuitive que tout lecteur peut faire d'un texte de Proust, de Beckett - ou de Duras. C'est du reste cette reconnaissance intuitive qui est à l'origine du pastiche ${ }^{30}$. Le style traverse et informe l'épiderme du tissu textuel (sinon d'ailleurs, il n'y aurait pas de plagiat possible), mais peut-il être aussi fonction de la mise en ceuvre scripturaire du texte ? des opérations qui ont présidé à sa genèse ? d'une méthode (personnelle ?) d'écriture ?

C'est la question - majeure pour une collaboration à venir - que soulève D. Alamargot, sans doute en guise d'invite : «Si le style se marque dans le texte produit, la question pour la psychologie cognitive est d'évaluer si et comment le style du texte est sous-tendu par un style dans les traitements. Si l'on admet que le souci permanent d'un écrivain est d'améliorer la qualité et l'originalité de son texte, alors il est probable qu'il ait instauré des enchaînements particuliers de processus caractérisant un stylé affirmé » (op. cit., p. 169).

Dans une démarche volontairement synthétique, nous avons présenté ici quelques concepts propres à la psychologie cognitive, possiblement exportables vers le champ de la critique génétique et tout particulièrement vers celui de stylistique génétique. En effet, nous avons donné une définition, et une illustration génétique - grâce à Leiris - de l'effet épistémique qui intervient lors de la textualisation, dans le cadre de ce que la psychologie appelle l'écriture libre ou mode romantique (l'écriture à déclenchement rédactionnel des généticiens). Cet effet épistémique s'observe en effet dans le processus catalytique de la phrase - laquelle en se formulant travaille la syntaxe et engendre de nouveaux contenus sémantiques. Pour paraphraser le proverbe, dans le cadre du mode romantique des psychologues, c'est en écrivant que l'on trouve quoi écrire. Nous avons également mis en commun la notion de style - style de genèse (lumineusement exploré par A. Herschberg Pierrot) et style processuel ou signature rédactionnelle que la psychologie définit comme un type d'enchaînement des sous-processus scripturaires propre à un individu. Si les typologies peuvent, à juste titre, rencontrer des réticences, elles peuvent tout de même servir de base de travail, et fournir des hypothèses que les corpus vérifient - ou non. Dans cette perspective, il conviendrait certainement d'interroger de nouveau la distinction entre scripteurs à déclenchement rédactionnel et scripteur à programme, en prenant notamment en compte la question du genre textuel pratiqué (et dans la démarche d'analyse des genres qui est pratiquée en analyse du discours). À cette distinction revisitée s'en ajouterait une autre (les deux étant évidemment intimement liées) : celle des profils de scripteurs au moment de la textualisation. Comme nous l'avons souligné, la textualisation obéit sans doute à des mécanismes différents si le scripteur a un plan préétabli (qu'il élabore lui-même ou qui est imposé par une schématisation héritée des pratiques sociale et de genres discursifs). Elle obéit sans doute aussi à des tendances au plan des opérations scripturaires - qui soit réduisent une première version, soit au contraire ne cessent de l'amplifier. L'étude approfondie de la genèse de la phrase longue (mais qu'est-ce qu'une phrase longue ?) ou des segments lapidaires de certaines œuvres pourraient nous renseigner sur cette syntaxe dynamique que donnent à voir l'ajout, la substitution, la suppression ou le déplacement.

29. D. Alamargot, « Du rédacteur à l'écrivain... », art. cit., p. 169.

30. Voir par exemple M. Duraille (alias P. Rambaud), Mururoa mon amour, Paris, Lattès, 1996 ; ou J.-F. Jeandillou, Supercheries littéraires. La vie et l' œuvre des auteurs supposés, Genève, Droz, 2001. 
On ne peut qu'insister - dans une démarche de prospective - sur la nécessité d'une étude pleinement interdisciplinaire sur la production des écrivains, c'est-à-dire sur la performance experte. Cette démarche interdisciplinaire impliquerait bien sûr la critique génétique littéraire mais aussi les sciences du langage et la psychologie cognitive ${ }^{31}$. Ce champ d'investigation peut se développer dans l'écriture électronique, directement observable, sans doute avec des modalités nouvelles propres au médium électronique (temps réel notamment) ${ }^{32}$. Ceci suppose d'abord une réflexion commune, que nous appelons de nos vœux, sur ce médium d'écriture et ce qu'il implique au plan des opérations scripturaires, des sous-processus et du traitement de l'information ${ }^{33}$. Comme le souligne P.-M. de Biasi, l'ère "tout numérique » de l'écriture est loin de signifier la fin de la critique génétique : " Numérique, par nature, les brouillons d'aujourd'hui ont une structure spontanément prête au calcul. Ils attendent seulement les machines qui sauront nous aider à les interpréter ${ }^{34}$. »

Si nous nous sommes interrogée sur les méthodes et les acquis théoriques à mettre en commun, nous voudrions - pour terminer - ouvrir sur la question des corpus. Mises à part des recherches suggestives lancées notamment sur la genèse des textes scientifiques, de l'architecture ou du cinéma ${ }^{35}$, la critique génétique s'est beaucoup consacrée - pour ce qui concerne le texte - à la production des textes littéraires, c'est-à-dire à la genèse des æeuvres, mais il conviendrait peut-être d'ouvrir le corpus textuel à d'autres genres de texte, comme les textes de presse ou le discours politique. Une ouverture semble s'imposer au-delà du clivage d'ailleurs flou entre écrivain/écrivant. En effet, l'analyse génétique et textuelle des discours et en particulier des discours non spécifiquement littéraires, permettrait de saisir la genèse de genres discursifs jusque-là restés à l'écart de l'approche génétique. On peut penser à la genèse de textes relevant de l'essai 36 ; on peut aussi évoquer la genèse des genres journalistiques, notamment du journalisme littéraire. Ou bien encore à celle des allocutions - qui convoquerait le travail des "plumes » - ainsi que la genèse de textes parlementaires : l'étude de la genèse d'un discours, comme celui que prononça R. Badinter, par exemple, pour l'abolition de la peine de mort, ne manquerait pas d'enrichir la critique génétique et son appareillage théorique ${ }^{37}$.

En clair, pour peu que l'on se donne le temps de construire une interdisciplinarité réelle, qui puisse mettre en commun les outils et les acquis théoriques des sciences du langage, de la critique génétique et de la psychologie cognitive - dont, rappelons-le, l'intérêt pour les processus de création va croissant -, c'est un espace considérable qui s'ouvre à l'investigation.

31. Sur les modalités de cette collaboration, voir S. Pétillon, «Style, critique génétique et modèles rédactionnels : perspectives linguistiques », dans Corpus, ${ }^{\circ}$ 5, « Corpus et stylistique », 2006, p. 39-73. Voir également l'ensemble des recherches menées dans le cadre du GDR 2657 «Production Verbale Écrite», dirigé par D. Alamargot et S. Plane.

32. Voir l'article très suggestif de C. Doquet-Lacoste, «L'objet insaisissable : l'écriture sur traitement de texte », Genesis, n 27, 2006, p. 35-43.

33. À ce titre, voir l'ensemble du numéro 27 de Genesis, 2006.

34. P.-M. de Biasi, «Le cauchemar de Proust », Médium, $\mathrm{n}^{\circ} 10$, «Le numérique en toutes lettres», 2007, p. 127-137.

35 . Voir les volumes de Genesis consacrés à l'architecture $\left(n^{\circ} 14,2000\right)$, à l'écriture scientifique $\left(\mathrm{n}^{\circ} 20,2003\right)$ et au cinéma $\left(\mathrm{n}^{\circ} 28,2007\right)$.

36. Voir S. Pétillon, " "Surgissement de la langue et en deçà textuel" : genèse de l'essai Qu'est-ce qu'écrire ? II, de B. Noël », La Licorne, « Style et Genèse », à paraître.

37. S. Pétillon, « Genèse d'un texte parlementaire politiquement correct. Abréviations, mémoire et textualisation : l'écriture en urgence des secrétaires analytiques », dans Écritures abrégées (notes, notules, messages, codes...), Paris, Ophrys, coll. « Bibliothèque des Faits de langue », 2004, p. 163-174. 
SAbine Pétillon est chargée de recherches à l'ITEM (CNRS-ENS). Linguiste, elle est plus particulièrement spécialisée dans la ponctuation, l'écrit et la génétique textuelle (Les Détours de la langue : étude sur la parenthèse et le tiret double, Peeters, Bibliothèque de l'Information grammaticale, 2002). Depuis son entrée à l'ITEM, elle s'est notamment engagée dans l'étude des manuscrits de Roland Barthes, de François Mauriac ou de Bernard Noël, ainsi que dans la dimension énonciative et syntaxique de la genèse de l'écriture. Par ailleurs, elle s'est formée, grâce à l'étude des textes procéduraux, à l'approche de la production verbale écrite telle que l'aborde la psychologie cognitive.

Sabine Pétillon, sabine.petillon@gmail.com

\section{Psychologie cognitive et production écrite : de l'effet «épistémique " à la catalyse}

Dans cet article, l'auteur propose un parallèle entre l'approche psycho-cognitive de la production écrite et le modèle qu'en a proposé la critique génétique. Notamment, il est question de voir en quoi l'écriture « à programme » rejoint (ou pas) ce que les psychologues appellent le schéma-driven text, et d'examiner également comment l'écriture dite « romantique» (disons « sans plan » dans la terminologie des psychologues) rejoint l'écriture à « déclenchement automatique » de la critique génétique. On propose également une application de ces problématiques à travers les manuscrits de Michel Leiris.

This article establishes a parallel between the psycho-cognitive approach to written production and the model proposed by genetic criticism. In particular, the object is to see how programmatic writing does (or does not) meet what psychologists call "schema-driven text". We will also examine how "romantic" writing (or, according to psychologists' terminology: "unstructured text") meets genetic criticism's automatically triggered writing. We will also see how this approach can be applied to Michel Leiris' manuscripts.

In dem Beitrag wird eine Parallele vorgeschlagen zwischen der psycho-kognitiven Analyse schriftlicher Produktionsprozesse und dem Analysemodell der ,critique génétique“. Insbesondere gilt es zu untersuchen, ob und inwieweit textorientiertes Schreiben mit dem von den Psychologen als „driven text" bezeichneten Schema zusammenhängt, und ob der von den Psychologen als „romantisches Schreiben“ oder als „Schreiben ohne Plan“ bezeichnete Prozess etwas zu tun hat mit dem prozessorientierten Schreiben der „critique génétique“. Eine praktische Anwendung dieser Fragestellungen bieten Handschriftenbeispiele von Michel Leiris.
En este artículo, el autor propone un paralelo entre el enfoque psico-cognitivo de la producción escrita y el modelo que ha elaborado al respecto la crítica genética. Se trata en particular de ver hasta qué punto la escritura "con programa" corresponde (o no) a lo que los psicólogos llaman el schema-driven text y de examinar de qué manera la escritura llamada "romántica" (o bien, "sin plan", en la terminología de los psicólogos) corresponde a la escritura con "arranque automático" de la crítica genética. Se propone también una aplicación de estas problemáticas a través de los manuscritos de Michel Leiris.

Neste artigo, a autora propõe um paralelo entre a abordagem psico-cognitiva da produção de escrita e o modelo da crítica genética. Trata-se de saber que medida a escrita "com programa" se aproxima (ou não) do que os psicólogos chamam schemadriven text, e de examinar igualmente como a escrita dita "romântica" (digamos "sem plano" na terminologia dos psicólogos) se aproxima da escrita de "arranque automático" da crítica genética. Uma aplicação destas problemáticas é feita sobre manuscritos de Michel Leiris.

Nel saggio, la studiosa propone un parallelo tra l'approccio psico-cognitivo della produzione scritta e l'approccio prospettato dalla critica genetica. Esaminando, soprattutto, gli elementi che permettono (o meno) di affermare che la scrittura "in base ad un programma" si accorda con lo schema-driven text, definito dagli psicologi, si riflette qui, al contempo, sui modi in cui la scrittura detta "romantica" (vale a dire "senza piano di lavoro", secondo la terminologia degli stessi psicologi) si accosta alla scrittura a "disinnesto automatico" della critica genetica. Viene, a supporto, presentata un'applicazione delle premesse metodologiche enunciate basata sugli autografi di Michel Leiris. 